\title{
Vol. 68, No. 42
}

In the report "Global Routine Vaccination Coverage, 2018," on page 937, in the list of authors, the fifth author should have been listed as Samir V. Sodha, MD. 